\title{
BMJ Open Combined effects of individual and neighbourhood socioeconomic status on older adults' mortality: a retrospective follow-up study in Hong Kong
}

\author{
Yingqi Guo, ${ }^{1,2}$ Kristy Shuk Ting Chan, ${ }^{2}$ Chee Hon Chan, ${ }^{1,2}$ Qingsong Chang, ${ }^{3}$ \\ Ruby SY Lee, ${ }^{4}$ Paul Siu Fai Yip (1D) ${ }^{1,2}$
}

To cite: Guo Y, Chan KST, Chan $\mathrm{CH}$, et al. Combined effects of individual and neighbourhood socioeconomic status on older adults' mortality: a retrospective follow-up study in Hong Kong. BMJ Open 2021;11:e043192. doi:10.1136/ bmjopen-2020-043192

\section{- Prepublication history and} additional supplemental material for this paper are available online. To view these files, please visit the journal online ().

Received 03 August 2020 Revised 24 March 2021 Accepted 26 March 2021

Check for updates

(C) Author(s) (or their employer(s)) 2021. Re-use permitted under CC BY-NC. No commercial re-use. See rights and permissions. Published by BMJ.

${ }^{1}$ Department of Social Work and Social Administration, The University of Hong Kong, Pokfulam, Hong Kong, China ${ }^{2}$ Hong Kong Jockey Club Centre for Suicide Research and Prevention, The University of Hong Kong, Hong Kong, China ${ }^{3}$ School of Sociology and Anthropology, Xiamen University, Fujian, China

${ }^{4}$ Elderly Health Service, Department of Health, The Government of the Hong Kong SAR, Hong Kong, China

Correspondence to Professor Paul Siu Fai Yip; sfpyip@hku.hk

\section{ABSTRACT}

Objectives This study examined the interaction effects of individual and neighbourhood socioeconomic status (SES) in older adults in Hong Kong, considering all-cause and cause-specific mortality from respiratory disease, cancer, cardiovascular diseases, ischaemic heart disease, stroke, nonmedical disease and suicide.

Design A retrospective follow-up study.

Setting Hong Kong Special Administrative Region, a rapidly ageing society with $16.1 \%$ residents aged 65 years or older in 2020.

Participants 43910 people aged 65 years or older were enrolled at baseline. They had participated in health check-ups during 2000-2003 in one of the Elderly Health Centres. Observation periods started on the date of the participant's first health check-up, and ended at death, or 31 December 2011, whichever occurred first.

Outcome measures All-cause and cause-specific mortality over the study timeframe.

Analysis Cox's proportional hazards regression models were applied to estimate the adjusted HRs of mortality, by including covariates at neighbourhood (deprivation) and individual levels (poverty, education and type of housing).

Results The 'double tragedy theory' (ie, lower SES persons living in lower SES neighbourhoods have worst health outcomes) was more related to cancer, while the 'psychosocial comparison theory' (ie, lower SES persons living in higher SES neighbourhoods have poorer health outcomes) was more related to cardiovascular, ischaemic heart disease, and stroke.

Conclusion There were important interaction effects between neighbourhood and individual factors on mortality. Policies based on the interaction between individual and neighbourhood SES should be considered. For instance, for cancer, targeted services (ie, free consultation, relevant treatment information, health check-up, etc) could be allocated in socioeconomically deprived areas to support individuals with low SES. On the other hand, more free public services to reduce psychological stresses (ie, psychological support services, recreational services, health knowledge information, etc) could be provided for those individuals with low SES living in higher SES areas to reduce stroke, cardiovascular and ischaemic heart diseases.
Strengths and limitations of this study

- This study tests a large data set captured from comprehensive medical records combined with comprehensive follow-up. This provided extensive information on all-cause and cause-specific mortality.

- An appropriate statistical analysis approach (Cox's survival model) was applied to calculate interaction effects of individual and neighbourhood socioeconomic status (SES).

- There was no information in the mortality data set on physical and social activities and, thus, the effects of these variables on mortality could not be explored,

- At the neighbourhood level, only SES was explored. Other aspects of neighbourhood (such as social and physical (built) characteristics) should be explored.

- This data set is now a decade old, and analysis should be undertaken of health check data collected since 2011 to determine whether neighbourhood and individual SES effects have changed over time.

\section{INTRODUCTION}

Individual socioeconomic inequality has long been linked to health outcomes. ${ }^{1-3}$ Individuals with lower socioeconomic status (SES) are more likely to suffer from poorer health status than people with higher SES. ${ }^{45}$ Moreover, SES inequality at neighbourhood level can also influence health outcomes. ${ }^{6}$ Those who live in socioeconomically deprived (low SES) areas have a higher probability of suffering poorer health outcomes than those living in higher SES areas. ${ }^{7}$ However, few studies have explored the combined effects of individual and neighbourhood SES on health outcomes, particularly in the elderly. ${ }^{7-9}$ Some studies suggest that poorer persons living in poorer neighbourhoods suffer the worst consequences (the 'absolute poverty' phenomenon). ${ }^{10}$ This is explained by the 'double tragedy' theory, where neighbourhood disadvantage amplifies the burdens on 
individuals living within it. Moreover, poorer neighbourhoods tend to be more disorderly, deprived of resources and have weaker bonds among individuals living within them, which in turn prevents already disadvantaged people to access the resources they need. ${ }^{11}$ However, other studies describe a different phenomenon, where poorer persons living in deprived areas experience better health outcomes than poorer persons living in nondeprived areas. ${ }^{12}$ This is the 'relative poverty' phenomenon, explained by the 'psychosocial comparison' theory. ${ }^{13}$ These theoretical inconsistencies highlight the need to test combined individual and neighbourhood SES effects, on mortality. Moreover, most studies to date have considered either overall mortality only or have explored mortality in only one disease. ${ }^{8} 14-18$ A more detailed study is indicated to investigate mortality patterns across different SES groups for common diseases.

Most of what is known about the relationship between SES, and health in older adults has come from Western societies, which are more economically developed than many Asian societies, and where social welfare systems are more established and comprehensive. Hong Kong is an Asian nation currently experiencing significant SES inequality, where the population is ageing quickly in terms of longer lifespans and increasing percentage of older people in the population. Furthermore, the majority of older adults in Hong Kong live without family support and increasingly need to rely on neighbourhood resources. It is, thus, important to understand the combined effects of individual and neighbourhood SES on their health as they age.

Compared with Western countries, Hong Kong presents a unique situation where public services are well provided in SES deprived areas (characterised by higher percentages of poor people). ${ }^{19}$ Therefore, we posit that the 'double tragedy' phenomenon reported in Western studies may not be found in Hong Kong, but rather the 'psychological comparison' theory might be more relevant to Hong Kong contexts. Rapid ageing with widening inequality is nonetheless not unique to Hong Kong, as it is common across many high-income East Asian countries and China. Therefore, a study conducted in Hong Kong might provide important evidence on which to frame policies to support healthy ageing in other Asia countries.

The aims of this study are to: (1) explore the combined effects of individual and neighbourhood SES on mortality and (2) compare patterns of disease-specific mortality across combined individual and neighbourhood SES subgroups. We tested the hypotheses that interaction between individual and neighbourhood SES can affect mortality, and that interaction patterns on mortality differ for different diseases.

\section{METHODS}

Study design

A retrospective follow-up study.
Patient and public involvement:

This study reports secondary analysis of a routinely collected deidentified data set.

\section{Data}

Baseline data came from health check-up information collected between 2000 and 2003 from 44607 older adults at their first enrolment in the Hong Kong elderly check-up system. Participants were aged 65 years at first assessment. Yearly assessments were conducted at one of the 18 elderly health centres in Hong Kong. Each assessment captured information on demographic and SES (age, gender, marital status, education, housing), lifestyle habits (history of smoking, alcohol consumption, physical activity), chronic diseases (self-reported chronic diseases which were then verified and supplemented by clinical diagnoses), functional status and mental health and survival. ${ }^{20}$ The observation period started on the date of the first health check-up and ended at death, or 31 December 2011, whichever occurred first. Participants with missing residential neighbourhood information were excluded from our data set, leaving a sample of 43910 older adults (reflecting 5.9\% of the total Hong Kong elderly population). Logistic regression models were constructed to test differences in age, gender, education and marital status between those who reported and did not report residential neighbourhood information, finding no significant differences. As the health check-up was funded by the Government and participation was voluntary, participants differed significantly in terms of age and gender from the overall elderly population living in the community in Hong Kong (online supplemental appendix table 1).

\section{Dependent variables}

The outcomes of interest were all-cause, and causespecific, mortality over the study period. Cause of death was coded using the International Classification of Diseases (ICD) V.9 or V.10. Cause of death included respiratory disease (ICD-9 460-519 or ICD-10 J00-98), cancer (ICD-9 140-208 or ICD-10 C00-D48), cardiovascular diseases (ICD-9 390-459 or ICD-10 I00-99), ischaemic heart disease (ICD-9 410-414 or ICD-10 I20-25), stroke (ICD-9 430-439 or ICD-10 I60-69), nonmedical (ICD-9 E800-990 or ICD-10 S00-Y98) and suicide (ICD-9 E950959 or ICD-10 X60-84).

\section{Individual SES}

Individual SES was measured by three variables, comprising poverty status (receiving Comprehensive Social Security Assistance ('CSSA')), education attainment level (illiterate, uneducated but can read and write a few words, primary, secondary or postsecondary) and type of housing (public and aided housing, rented private housing, self-owned private housing, others such as institutions, temporary housing, etc). In Hong Kong, the CSSA is a means-tested subsidy for those in financial need. ${ }^{21}$ 


\section{Neighbourhood SES}

Five items characterised neighbourhood SES: (1) the proportion of people in the neighbourhood with nonprofessional jobs, (2) the proportion of people in the neighbourhood with secondary education or below, (3) unemployment rate, (4) the proportion of overcrowded households and (5) poverty rate, calculated using the neighbourhood deprivation index in Hong Kong ( $\mathrm{Hsu}, 2015)$. The neighbourhood deprivation index was calculated at both the smaller geographical scale (ie, Large Street Blocks ('LSB's)) and the larger geographical scale (ie, Small Tertiary Planning Units ('STPU's) ) level by merging these five basic indicators. The LSBs and the STPUs are geographical units covering all small areas in Hong Kong. Z-scores at the LSB and the STPU levels were calculated using the mean and SD of the five indicators. The z-score was calculated by subtracting the mean from the observed value for each indicator and dividing this by the SD. The five standardised z-scores were then summed. The 'deprived neighbourhoods' and 'nondeprived neighbourhoods' were distinguished using the median neighbourhood deprivation index.

\section{Covariates}

A number of covariates were added to models testing all-cause and cause-specific mortality risk and SES status. These comprised age in years (65-69, 70-74, 75+), sex (male, female), marital status (never married, married, widowed, separated/divorced), smoking status (currentsmoker, ex-smoker, never-smoker), alcohol consumption status (never drink, drank before but quit already, social drinker/seasonal drinker, regular drinker), depressive symptoms and functional capacity. ${ }^{22} 23$ Depressive symptoms were measured by the Chinese V.15-item Geriatric Depression Scale, and depression was distinguished using a cut-off score of eight following previous studies. ${ }^{24}$ Functional capacity was measured by activities of daily living as continuous variable. $^{25}$

\section{Statistical analysis}

Survival analysis was used to estimate the associations between mortality outcomes and SES status (combining individual and neighbourhood levels) over the observation period. Cox's proportional hazards regression models with covariate adjustment were applied. This is a regression model commonly used for investigating the association between survival time and one or more predictors. ${ }^{26}$ Three sets of regression models, each with one type of individual SES indictor as mentioned above (ie, poverty, education, types of housing), were included in this study. The proportional hazard assumptions were tested by using Schoenfeld residuals and Kaplan-Meier plots, and no violations were found. The analyses were repeated with all-cause mortality and cause-specific mortality to explore potential variations.

\section{RESULTS}

\section{Descriptive statistics}

There were between 8 and 11 years from initial assessment to final date of follow-up, averaging approximately
10 years. Of the total sample, $9754(22.2 \%)$ died during the observation period (34169 (77.8\%) survived until 31 December 2011). Of the deaths, there were 1841 (18.9\%) from respiratory diseases, $3423(35.1 \%)$ from cancer, $2675(27.4 \%)$ from cardiovascular diseases, $1045(10.7 \%)$ from ischaemic heart disease, $904(9.3 \%)$ from stroke, $258(2.6 \%)$ from nonmedical mortality and $108(1.1 \%)$ from suicide. Considering all deaths, only $10 \%$ had only one disease. There were significantly higher all-cause mortality rates for men; participants older than 75 years; those who were alone (never married, or were widowed, separated or divorced); those with low income; those with lower educational attainment; those living in institutions and public housing and those living in deprived areas (see table 1).

\section{Predictors of all-cause mortality}

Figure 1 reports the separate individual and neighbourhood SES risks for all-cause mortality after controlling for all covariates. For individual SES, those who were poor, with lower education attainment, who lived in public housing, or rented in private housing, or lived in institutions, had significantly higher mortality risk than the rest of the sample. Considering neighbourhood SES, those who lived in deprived areas had significantly higher risk of dying. The results were consistent at the smaller geographical scale (ie, LSB) as well as the larger geographical scale (ie, STPU). Figure 2 reports the combined results of the individual and neighbourhood SES after controlling for all covariates. Poor participants living in nondeprived areas had an elevated risk of dying compared with poor persons living in deprived areas, however, those who were not poor and lived in nondeprived areas had the lowest risk. Compared with other education neighbourhood deprivation groups, those who had postsecondary education but lived in deprived areas had similar poor mortality risk to those with primary or below education, who lived in deprived neighbourhoods. Those who lived in institutions in deprived areas had the poorest rate of survival compared with other groups.

\section{Predictors of cause-specific mortality}

The findings from the smaller and larger geographical scales were consistent (ie, in terms of positive or negative effects) with generally stronger effects at smaller geographical scale (ie, in terms of HRs). Therefore, only the results from the smaller geographical scale are reported here. Five disease-specific causes of mortality (respiratory disease, cancer, cardiovascular disease, ischaemic heart disease and stroke) showed significant differences across the combined individual and neighbourhood SES groups after adjusting for all covariates. The remaining disease-specific mortality causes (nonmedical, suicide) had similar risks in all combined individual and neighbourhood SES groups. Therefore, only the results from these two diseases are reported in the table (online supplemental appendix table 2), rather than as visuals (online supplemental appendix figure 1). 
Table 1 Baseline characteristics of sample from 2000 to 2003

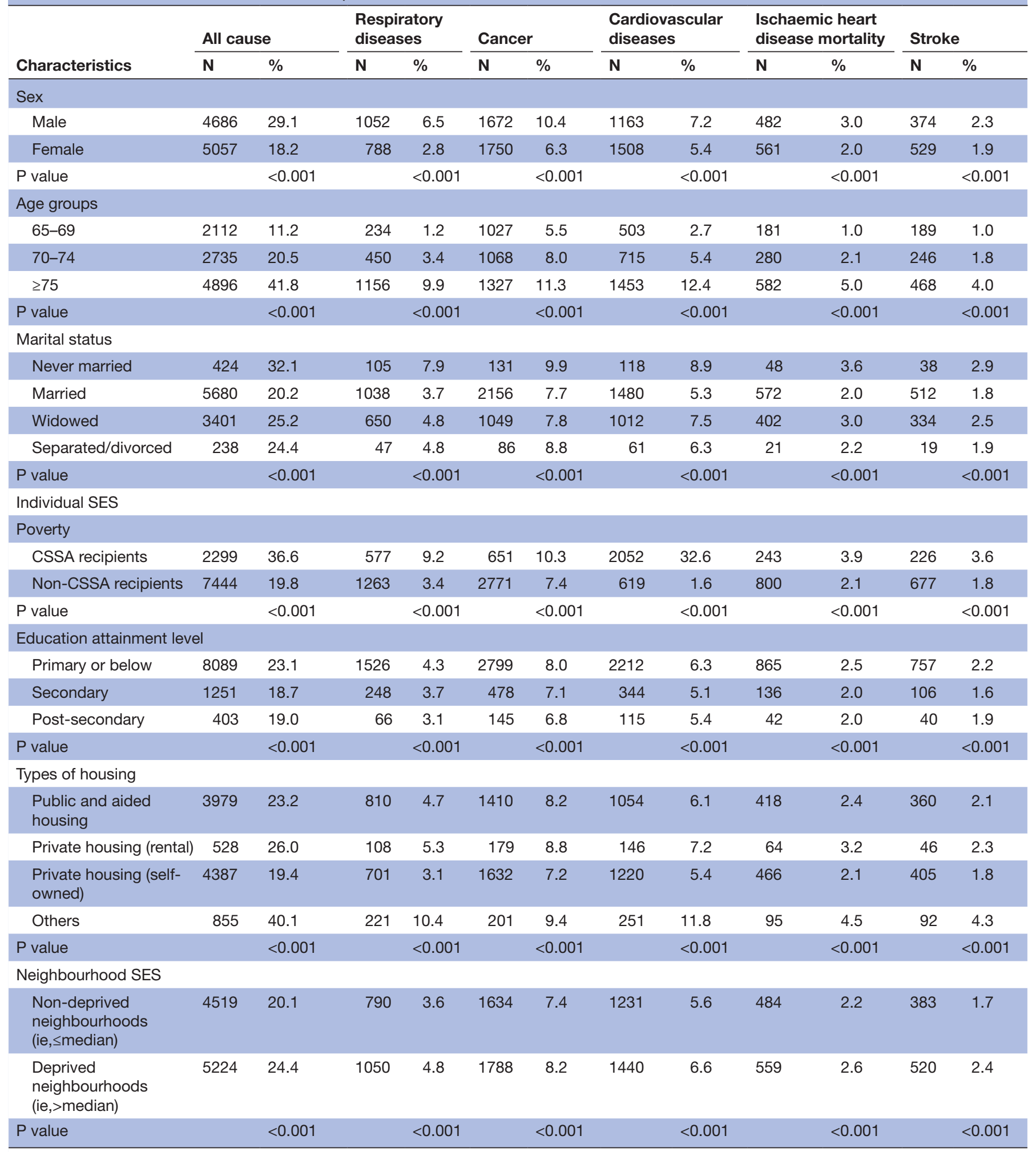

CSSA, Comprehensive Social Security Assistance; SES, socioeconomic status.

Regarding individual poverty and neighbourhood deprivation status, all disease types showed similar risk patterns except for cancer, where poor people living in deprived areas had the highest mortality risk. For the other four disease types, poor persons living in deprived areas had lower mortality risk than poor persons who lived in nondeprived areas. Regarding individual education and neighbourhood deprivation, unique patterns were found for cardiovascular diseases, ischaemic heart disease and stroke, where people in nondeprived areas who had 

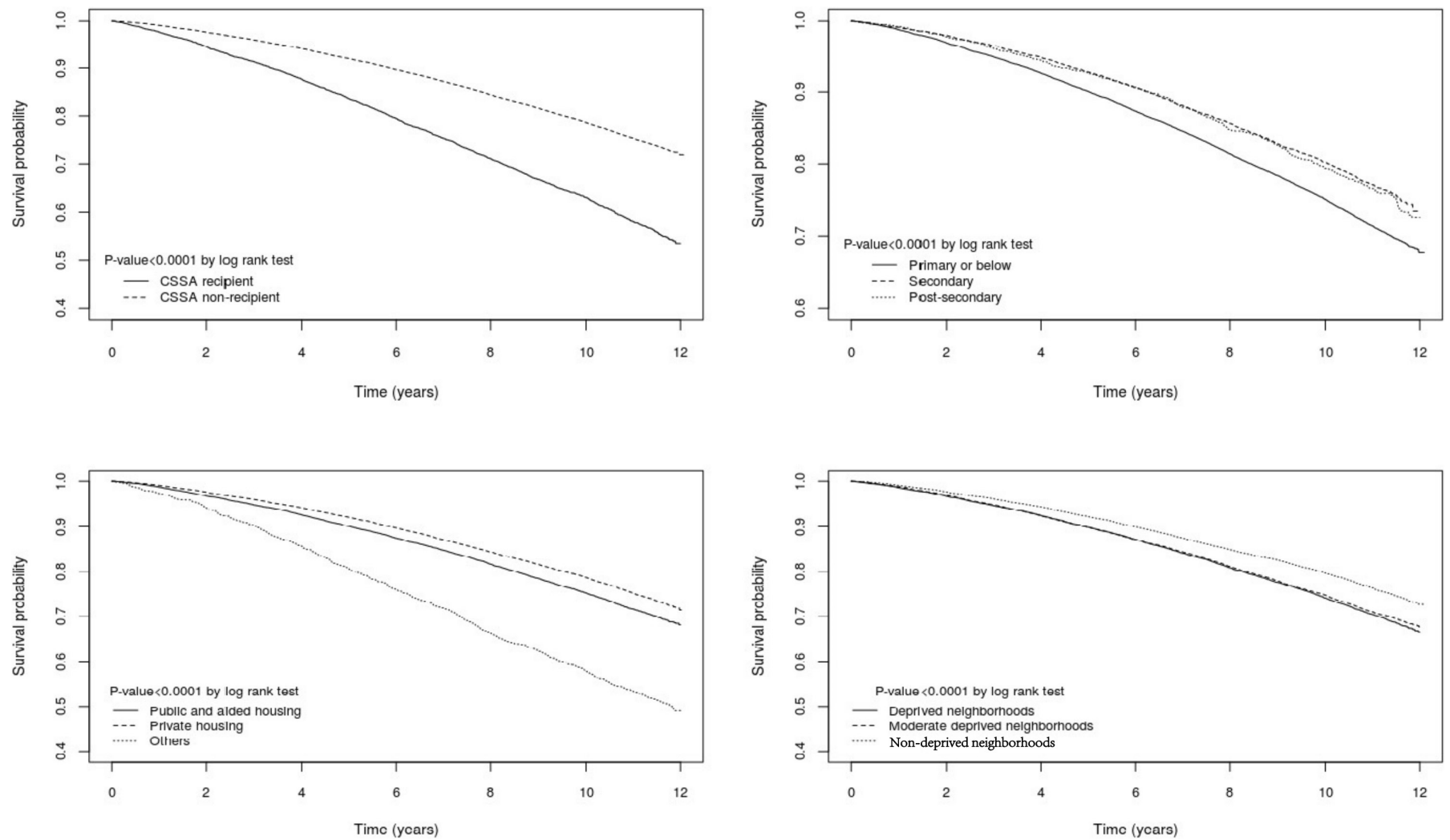

Figure 1 Survival probability for individual SES (ie, poverty, education, types of housing) and neighbourhood (ie, LSB) SES (ie, neighbourhood deprivation). LSB,Large Street Block; SES, socioeconomic status.

higher education levels also had higher mortality risks. However, postsecondary education was protective of high mortality risk for those who lived in deprived areas. Regarding individual housing type and neighbourhood deprivation, cancer showed a distinctly different patterns compared with the other five disease types (which were all similar). For cancer deaths, those who lived in institutions and in deprived areas had significantly higher mortality risk, however, for the other five types of diseases, those who lived in institutions and in nondeprived areas, had highest mortality risk.

\section{DISCUSSION}

This paper provides important and new information on the impact of individual and neighbourhood SES on mortality risk in Hong Kong between 2000 and 2011. The findings were generated from a very large, comprehensive data set and will assist healthcare providers to develop health promotion interventions for healthy ageing, specific to local contexts. The Hong Kong population is not only rapidly ageing, but more people are living for longer. Hong Kong is a very large, busy city with high density housing in a range of SES locations. ${ }^{27}$ With more people living in such densely populated neighbourhoods, individual and neighbourhood SES is bound to significantly impact health. This study found evidence for application of both theories relevant to specific diseases, thus we rejected our hypothesis that the double tragedy theory would not be found in this HK dataset. The absolute poverty theory (double tragedy) appears to be more related to cancer than other diseases. This study suggests that cancer mortality can be explained by it, where vulnerable persons (poor and living in institutions) who reside in deprived neighbourhoods are in the worst situation for mortality risk. However, the other disease types generally tended to fall within the "psychosocial comparison' pattern because poor persons who lived in deprived neighbourhoods were in a better situation than poor persons who lived in nondeprived areas. This may be explained that poor persons who lived in nondeprived neighbourhoods may put themselves under increased stress by comparing themselves with their richer neighbours. Moreover, Hong Kong has the unique phenomenon that poorer neighbourhoods are better provided for, than richer neighbourhoods, with public services. ${ }^{28}$ The lack of public resources in the nondeprived areas may further hinder those poor persons in these areas from accessing public medical services. However, in this situation, the 'double tragedy phenomenon (individual poor and nondeprived neighbourhood lack of services)' theory may also explain this finding.

\section{Cancer-specific mortality}

Compared with other diseases, cancer is more lethal and requires greater accessibility to better-funded resources. ${ }^{29}$ Cancer patients with low individual SES tend to seek medical advice or undergo treatment in public 


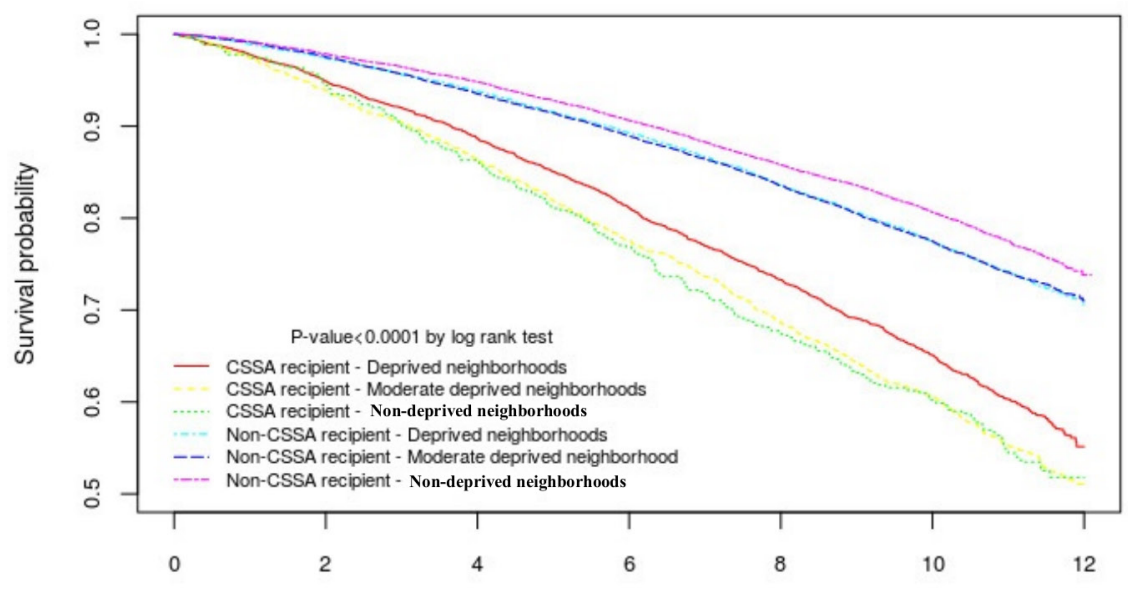

Time (years)
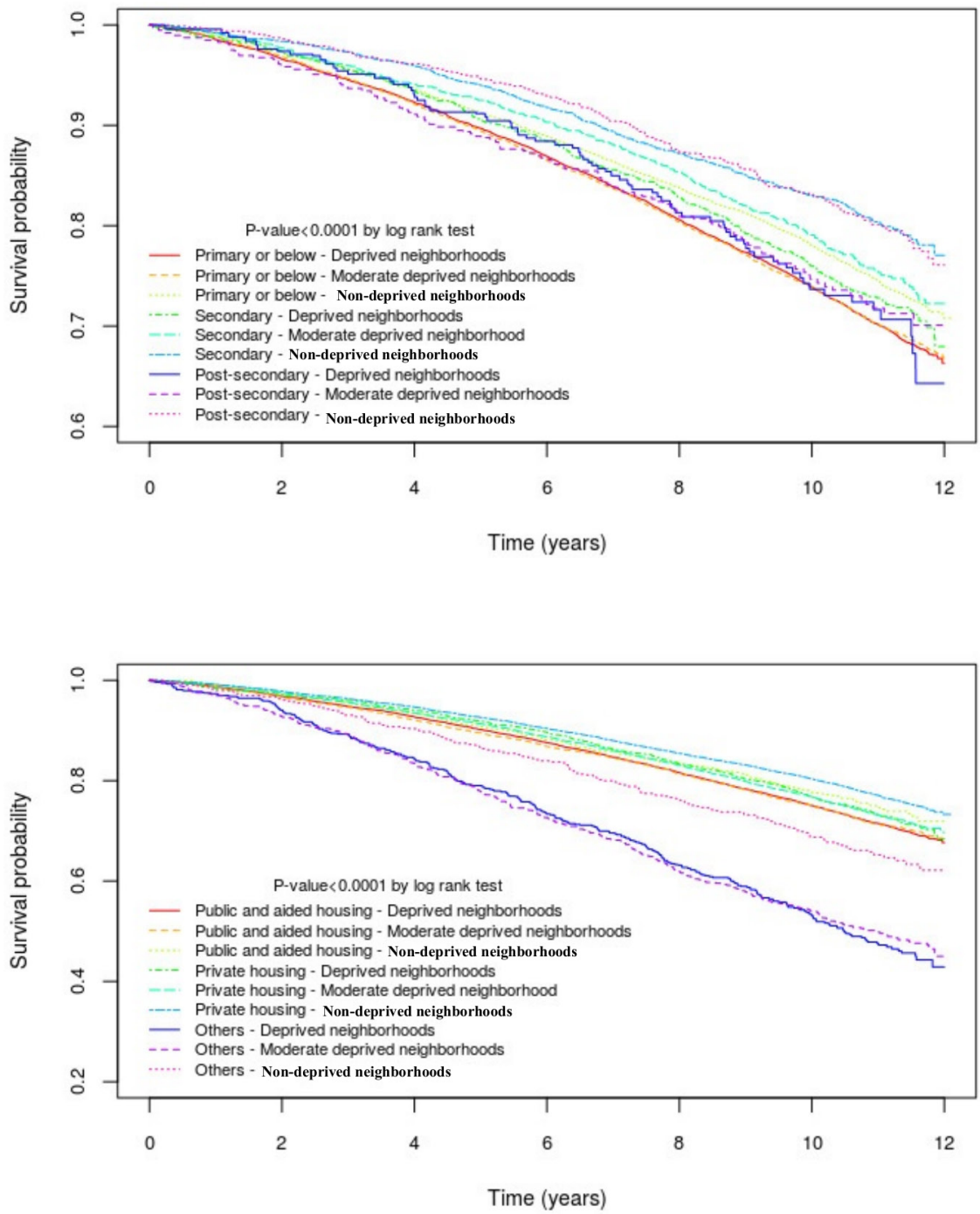

Figure 2 Survival probability for all-cause mortality. CSSA, Comprehensive Social Security Assistance.

hospitals, as the private hospitals are expensive. However, the waiting time in public hospitals is far longer than in private hospitals, therefore, poor persons on a waiting list might be disadvantaged because they are not offered optimal treatment due to the delay. ${ }^{30}$ Moreover, the cost of some services in the public system still needs to be 
borne by patients. Accessibility of private medical services in deprived areas was generally poor, although these areas were well provided for the public sector services. Accessing care in private medical centres and hospitals can reduce waiting times for surgery, reconstruction, radiotherapy and chemotherapy, and these services are mostly located in nondeprived areas. ${ }^{19}$ Therefore, even if the individual SES is not low, difficulties accessing private services may reduce their availability for timely prediagnosis and treatment. Moreover, cancer patients with higher SES and those living in more advantaged neighbourhoods may have more opportunity to improve their health with better finances, power, social connections ${ }^{31}$ and knowledge. In contrast, the greater isolation of individuals, with low SES who live in deprived areas with less collective agency, efficacy and social support, may make it more difficult for them to obtain community support and useful advice. $^{32}$

\section{Cardiovascular-specific, ischaemic heart disease-specific, stroke-specific mortality}

For cancer and respiratory disease-specific mortality, higher education attainment is a protective factor irrespective of individuals' neighbourhood SES. However, for cardiovascular, ischaemic heart disease and stroke, higher education attainment is protective only for persons who live in deprived areas. An explanation for this may be that compared with those with lower education attainment, higher levels of education attainment provides people with access to more information, knowledge and awareness of available resources to keep healthy and improve health status. Moreover, the well-provided public services in deprived areas may also improve people's access to the facilities for physical and social activities. ${ }^{19}$ For those higher educated persons living in nondeprived areas, the potential reason that education is not a protective factor might be related to the so-called 'disease of affluence', which has previously been called diseases of the rich, for example, cardiovascular disease arising from fatty diets and lack of exercise. These diseases are in contrast to the so-called 'diseases of poverty', which largely result from, and contribute to, human impoverishment. In this study, the finding that educational attainment was not a protective factor in non-deprived areas may be explained by lifestyle factors, such as using private cars rather than public transportation and stressful lifestyles. Moreover, in Hong Kong, in the nondeprived areas, the public recreational services are not as good as those in deprived areas, therefore, less access to these services can also reduce older adults' chances of walking, and exercising regularly, irrespective of the fact that people with higher educational attainment would be more aware that keeping active can keep them healthy.

\section{Strengths and limitations}

This study tested a large data set of standard data items collected from older individuals at their yearly health checks in Hong Kong. It applied a robust statistical test
(Cox's survival analysis), which can yield good statistical power. Both all-cause mortality and cause-specific mortality were explored, on both smaller and larger geographical scales, all of which provide important information for future planning for health ageing in Hong Kong. However, there are limitations that constrain interpretation of findings. First, there was no information on physical and social activities in the health check, and these are important factors for health status. Second, at neighbourhood levels, only neighbourhood SES was explored, and we recommend that other aspects of neighbourhood (such as social and physical (built) characteristics) should be explored in future studies. Third, the study is of older adults who voluntarily participated in health examinations a decade or more ago. These participants may have a higher level of both physical and mental health than those who did not receive the health examinations. Fourth, the impact of SES status in the last decade may have changed, and, therefore, update analysis of health check data sets is required.

\section{CONCLUSION}

There were interaction effects between neighbourhood and individual factors on all-cause and disease-specific mortality, with different patterns across specific-cause mortality. The findings of this study suggest that health promotion policies should reflect health disparities among the combined individual and neighbourhood SES groups. Different mortality and risk patterns from different disease causes suggest that public health policies should be designed for specific diseases. For example, more cancer-related services (ie, free consultation, relevant treatment information, health check-up, etc) should be allocated to deprived areas to support disadvantaged persons. More public-free services (ie, psychological supporting services, recreational services, health knowledge information, etc) should be provided for people with lower SES who live in nondeprived areas. There are still large gaps between the poor and nonpoor in Hong Kong society, and Government should, therefore, pay more attention to policies that can help to reduce health inequalities and differences in mortality outcomes among different SES groups. The study findings provide insights that will assist other Asian countries undergoing similar process as Hong Kong (Taiwan, Japan, South Korea, Singapore).

Acknowledgements The authors are grateful for the constructive comments from the reviewers, the funding support from GRF grants $(1062000308,106160261)$ of the Research Grants Committee (RGC), Hong Kong. Gratitude to the Department of Health, Census and Statistics Department, Lands Department and Planning Department, the Government of the Hong Kong Special Administrative Region for their support in allowing access to the data. Appreciation also to Dr. Mengni Chen and Mrs. Esther Lee from the Hong Kong Jockey Club Centre for Suicide Research and Prevention, The University of Hong Kong for their vibrant discussions.

Contributors YG, CHC, PSFY conceived and designed the study. CHC gathered data, and RSYL, PSFY facilitated access to data. YG, KSTC and QC performed data analyses, and CHC supervised the analyses. YG drafted the manuscript. KSTC, CHC, 
QC, RSYL and PSFY revised the manuscript for important intellectual content. PSFY is the guarantor.

Funding This work was supported by General Research Fund (GRF) (Number 106160261) of the Research Grants Committee (RGC), Hong Kong. Gratitude to the Department of Health, Census and Statistics Department, Lands Department and Planning Department, the Government of the Hong Kong Special Administrative Region for their support in allowing access to the data. Appreciation also to Dr. Mengni Chen and Mrs. Esther Lee from the Hong Kong Jockey Club Centre for Suicide Research and Prevention, The University of Hong Kong for their vibrant discussions.

Competing interests None declared.

Patient consent for publication Not required.

Ethics approval This study was approved by Human Research Ethics Committee for Non-Clinical Faculties (HRECNCF) Committee (No. EA1501073), The University of Hong Kong.

Provenance and peer review Not commissioned; externally peer reviewed.

Data availability statement Data may be obtained from a third party and are not publicly available. Interested researchers can obtain the data by request or purchase from the Hong Kong Government by contacting relevant DepartmentsElderly Health Services of the Department of Health for elderly checkup data, Census and Statistics Department for the census tracts data, Lands Department for the Geo-Community Database and the GeoReference Database and Planning Department for the geographical census boundary data.

Supplemental material This content has been supplied by the author(s). It has not been vetted by BMJ Publishing Group Limited (BMJ) and may not have been peer-reviewed. Any opinions or recommendations discussed are solely those of the author(s) and are not endorsed by BMJ. BMJ disclaims all liability and responsibility arising from any reliance placed on the content. Where the content includes any translated material, BMJ does not warrant the accuracy and reliability of the translations (including but not limited to local regulations, clinical guidelines, terminology, drug names and drug dosages), and is not responsible for any error and/or omissions arising from translation and adaptation or otherwise.

Open access This is an open access article distributed in accordance with the Creative Commons Attribution Non Commercial (CC BY-NC 4.0) license, which permits others to distribute, remix, adapt, build upon this work non-commercially, and license their derivative works on different terms, provided the original work is properly cited, appropriate credit is given, any changes made indicated, and the use is non-commercial. See: http://creativecommons.org/licenses/by-nc/4.0/.

ORCID iD

Paul Siu Fai Yip http://orcid.org/0000-0003-1596-4120

\section{REFERENCES}

1 Hosseinpoor AR, Stewart Williams JA, Itani L, et al. Socioeconomic inequality in domains of health: results from the world health surveys. BMC Public Health 2012;12:198.

2 Kawachi I, Kennedy BP. Socioeconomic determinants of health : Health and social cohesion: why care about income inequality? BMJ 1997;314:1037.

3 Lago S, Cantarero D, Rivera B, et al. Socioeconomic status, health inequalities and non-communicable diseases: a systematic review. $Z$ Gesundh Wiss 2018;26:1-14.

4 Adler NE, Ostrove JM. Socioeconomic status and health: what we know and what we don't. Ann N Y Acad Sci 1999;896:3-15.

5 Mirowsky J. Education, social status, and health. Routledge, 2017.

6 Mitchell RJ, Richardson EA, Shortt NK, et al. Neighborhood environments and socioeconomic inequalities in mental well-being. Am J Prev Med 2015;49:80-4

7 Meijer M, Röhl J, Bloomfield K, et al. Do neighborhoods affect individual mortality? A systematic review and meta-analysis of multilevel studies. Soc Sci Med 2012;74:1204-12.

8 Shin J, Cho KH, Choi Y, et al. Combined effect of individual and neighborhood socioeconomic status on mortality in patients with newly diagnosed dyslipidemia: a nationwide Korean cohort study from 2002 to 2013. Nutr Metab Cardiovasc Dis 2016;26:207-15.

9 Chang T-S, Chang C-M, Hsu T-W, et al. The combined effect of individual and neighborhood socioeconomic status on nasopharyngeal cancer survival. PLoS One 2013;8:e73889.

10 Jargowsky PA. Poverty and place: Ghettos, barrios, and the American City. Russell Sage Foundation, 1997.

11 Guo Y. Neighborhood poverty and wellbeing in Hong Kong: a spatial and multilevel analysis. HKU Theses Online (HKUTO), 2017.

12 Hsu C-Y, Chang S-S, Yip P. Individual-, household- and neighbourhood-level characteristics associated with life satisfaction: a multilevel analysis of a population-based sample from Hong Kong. Urban Studies 2017;54:3700-17.

13 Cheung F, Lucas RE. Income inequality is associated with stronger social comparison effects: the effect of relative income on life satisfaction. J Pers Soc Psychol 2016;110:332-41.

14 Winkleby M, Cubbin C, Ahn D. Effect of cross-level interaction between individual and neighborhood socioeconomic status on adult mortality rates. Am J Public Health 2006;96:2145-53.

15 Shin J, Choi Y, Kim SW, et al. Cross-level interaction between individual socioeconomic status and regional deprivation on overall survival after onset of ischemic stroke: National health insurance cohort sample data from 2002 to 2013. J Epidemiol 2017;27:381-8.

16 Turrell G, Kavanagh A, Draper G, et al. Do places affect the probability of death in Australia? A multilevel study of area-level disadvantage, individual-level socioeconomic position and all-cause mortality, 1998-2000. J Epidemiol Community Health 2007;61:13-19.

17 Dahl E, Ivar Elstad J, Hofoss D, et al. For whom is income inequality most harmful? A multi-level analysis of income inequality and mortality in Norway. Soc Sci Med 2006;63:2562-74.

18 Hagedoorn $\mathrm{P}$, Vandenheede $\mathrm{H}$, Vanthomme K, et al. A cohort study into head and neck cancer mortality in Belgium (2001-11): are individual socioeconomic differences conditional on area deprivation? Oral Oncol 2016;61:76-82.

19 Guo Y, Chang S-S, Chen M, et al. Do poorer areas have poorer access to services in Hong Kong? A small-area analysis based on multiple spatial accessibility indicators. Soc Indic Res 2018;138:1-21.

20 Shen C, Schooling CM, Chan WM, et al. Self-Rated health and mortality in a prospective Chinese elderly cohort study in Hong Kong. Prev Med 2014;67:112-8.

21 Yip $\mathrm{P}$, Chan M, So BK, et al. A decomposition analysis to examine the change in the number of recipients in the comprehensive social security assistance (CSSA) system. China Popul Dev Stud 2020:284-97.

22 Murray CJ, Lopez AD. Global mortality, disability, and the contribution of risk factors: global burden of disease study. Lancet 1997;349:1436-42.

23 Schulz R, Beach SR, Ives DG, et al. Association between depression and mortality in older adults: the cardiovascular health study. Arch Intern Med 2000;160): :1761-8.

24 Nyunt MSZ, Fones C, Niti M, et al. Criterion-based validity and reliability of the geriatric depression screening scale (GDS-15) in a large validation sample of community-living Asian older adults. Aging Ment Health 2009;13:376-82.

25 Bucks RS, Ashworth DL, Wilcock GK, et al. Assessment of activities of daily living in dementia: development of the Bristol activities of daily living scale. Age Ageing 1996;25:113-20.

26 Fox J. Cox proportional-hazards regression for survival data. An $R$ and S-PLUS companion to applied regression, 2002.

27 Monkkonen P, Zhang X. Socioeconomic segregation in Hong Kong: spatial and ordinal measures in a high-density and highly unequal City, 2011.

28 Kandt J, Chang S-S, Yip P, et al. The spatial pattern of premature mortality in Hong Kong: how does it relate to public housing? Urban Stud 2017;54:1211-34.

29 Folkman J, Kalluri R. Cancer without disease. Nature 2004;427:787.

30 Guttmann A, Schull MJ, Vermeulen MJ, et al. Association between waiting times and short term mortality and hospital admission after departure from emergency department: population based cohort study from Ontario, Canada. BMJ 2011;342:d2983.

31 Lin N. Inequality in social capital. Contemp Sociol 2000;29:785-779.

32 Guo Y, Chan $\mathrm{CH}$, Chang Q, et al. Neighborhood environment and cognitive function in older adults: a multilevel analysis in Hong Kong. Health Place 2019;58:102146. 